\title{
"EVALUATION OF INTRAVENOUS IRON VERSUS ORAL IRON IN MANAGEMENT_OF IRON DEFICIENCY ANEMIA IN PREGNANCY WITH SPECIFIC REFERENCE TO BODY IRON STORE"
}

Richa Bhargava, Manju Maheshwari

1. PG Resident, Department of Obstetrics \& Gynaecology, Mahatma Gandhi Medical College, Sitapura, Jaipur.

2. Professor \& Unit Head. Department of Obstetrics \& Gynaecology, Mahatma Gandhi Medical College, Sitapura, Jaipur.

\section{CORRESPONDING AUTHOR:}

Dr. Richa Bhargava,

C-37 Piyush Path,

Bapunagar, Jaipur, Rajasthan.

E-mail: thanviricha@gmail.com

ABSTRACT: INTRODUCTION: Anemia is the most common Nutritional deficiency disorder in the World. Iron-deficient anemia (IDA) is responsible for $95 \%$ of anemia during pregnancy. Parenteral iron is a useful treatment, although iron dextran use decreased due to anaphylaxis. Iron sucrose is a newer agent that has overcome the shortcomings of iron dextran. OBJECTIVE: The aim of this study was to compare the efficacy and tolerance of intravenous iron sucrose (IVIS) therapy with oral iron (OI) therapy in pregnant women with IDA with specific emphasis on body iron stores. MATERIALS AND METHODS: This prospective, randomized clinical trial included 100 pregnant women between < 32 weeks with established IDA who were treated with IVIS or OI (ferrous ascorbate). All patients were monitored for laboratory response and adverse effects. Independent sample- $t$ test was used for statistical analysis. $P<0.05$ was considered significant. RESULTS: Although hemoglobin increased in both the groups, increase in the reticulocyte count and percentage increase in hemoglobin was significantly higher in the IVIS group than in the OI group. Serum ferritin was significantly higher in the IVIS group than in the OI group $(P=0.000)$. The IVIS group had no major side-effects. Compliance was good with OI, although majority had gastrointestinal side-effects. CONCLUSION: IVIS is safe and effective in the treatment of IDA during pregnancy. Iron stores increased better with IVIS compared with OI.

KEYWORDS: Intravenous iron sucrose, iron deficiency anemia, oral iron, serum ferritin.

INTRODUCTION: Anemia is the most common Nutritional deficiency disorder in the World. WHO has estimated prevalence of anemia in developed and developing countries in pregnant women is 14 percent in developed and 51 percent in developing countries and 65-75 percent in India1.

About one third of global population (over 2 billion) are anemic ${ }^{2}$

Prevalence of anemia in all groups is higher in India as compared to other developing countries ${ }^{1}$.

Anemia is the most common indirect cause of maternal mortality in India. Prevalence of anemia among pregnant women in India is $57 \% 3$.

Iron-deficient anemia (IDA) is responsible for $95 \%$ of anemia during pregnancy ${ }^{4}$. 
The World Health Organization (WHO) defined anemia in pregnancy as hemoglobin values $<11 \mathrm{~g} / \mathrm{dl}^{5}$, and Centre for Disease Control (CDC) as a hemoglobin level $<11 \mathrm{~g} / \mathrm{dl}$ during the first and third trimesters and $<10.5 \mathrm{~g} / \mathrm{dl}$ during second trimester6.

Iron deficiency anemia remains the commonest medical disorder in pregnancy in the developing world 7 -11, with the burden of disease impacting on both the mother and the newborn (and subsequent child and later adult)

The odds ratio for low birth weight are tripled, while those for preterm delivery more than doubled in association with IDA $^{12}$.Anaemia and iron deficiency are associated with large placental weight and a high ratio of placental weight to birth weight (placental ratio) ${ }^{13}$, both of which are predictors of adult hypertension ${ }^{14}$.

Iron deficiency anemia has been defined by the National Academy of Sciences panel on nutrition and pregnancy ${ }^{15}$ as serum Ferritin level lower than 12 microgram/l.

Parenteral iron is a useful treatment, although iron dextran use decreased due to anaphylaxis. Iron sucrose is a newer agent that has overcome the shortcomings of iron dextran.

Iron sucrose complex is a more recent drug which is used intravenously for correction of IDA with less side effects and cost effectiveness. Clinical trials and the long history of use of iron sucrose injection worldwide have established efficacy and safety of this drug in patients with IDA; it is metabolically available very quickly after administration besides being safe, convenient, and more effective than intramuscular therapy in the treatment of IDA during pregnancy ${ }^{16}$.

The Intravenous iron therapy can replace blood transfusion in antenatal period for moderate IDA as there are numerous hazards of blood transfusion including transfusion of wrong blood, infection, anaphylaxis, and lung injury any of which would be devastating for mother ${ }^{17}$.

Recent evidence suggests that iron sucrose can be detected in high levels in liver circulation and marrow within 5 min after intravenous administration ${ }^{16}$.

Also, the accumulation of iron sucrose in organic parenchyma is much lower compared with iron-dextrans and iron-gluconate ${ }^{18}$.

Thus, Iron sucrose has revolutionized anemia management in pregnancy.

MATERIALS AND METHODS: The objectives of the study were to compare the efficacy and tolerance of intravenous iron sucrose (IVIS) therapy with OI therapy in pregnant women with IDA with specific emphasis on body iron stores.

This was a prospective study where 100 anemic antenatal women with hemoglobin 7-9 $\mathrm{g} / \mathrm{dl}$, mean corpuscular volume $<78 \mathrm{fl}$ and serum ferritin $<20 \mathrm{mcg} / \mathrm{l}$, Cases were randomly divided into two groups:

\section{a. Intravenous iron group \\ b. Oral iron group}

Both groups consisted of 50 patients each selected on random basis.

After detailed history and examination, laboratory investigations performed were hemoglobin, packed cell volume (PCV), red cell count, red cell indices, reticulocyte count and peripheral smear. IDA was confirmed by serum iron profile consisting of serum ferritin, serum iron and total iron binding capacity

Deworming was done in all patients before starting iron therapy. 
Tab. Mebendazole $100 \mathrm{mg}$ twice daily for three consecutive days.

Total dose of iron to be administered was calculated from the following formula:

(Target hemoglobin-patient's hemoglobin) $\times$ patient's weight in $\mathrm{kg} \times 2.21)+1000 \mathrm{mg}=$ mg of iron needed, where hemoglobin is taken as $11 \mathrm{~g} / \mathrm{dl}^{19}$.

In intravenous iron sucrose group calculated dose to be given as $200 \mathrm{mg}$ of iron to be dissolved in $200 \mathrm{cc}$. normal saline in one sitting and remaining doses were given on alternate days with maximum $600 \mathrm{mg}$ in a week. Infusions were given as outpatient basis with facilities of acute emergency care.

Doses were rounded off for convenience as ampoules are available in 50 and $100 \mathrm{mg}$ strength.

Initial $20 \mathrm{ml}$ was given slowly to look for any sign of intolerance- nausea, hypotension, headache, vomiting.

Folic acid $5 \mathrm{mg} /$ day was given to both groups.

Oral iron was withheld in intravenous group during therapy but was restarted after 2 weeks.

In oral iron group, Ferrous ascorbate tablet (each tab containing $100 \mathrm{mg}$ elemental iron). Number of tablets were decided according to calculated dose .

Patients were asked to note compliance on a calendar.

Both groups were instructed to eat high calorie and protein diet .

Two weeks after completion of therapy all laboratory test were repeated and statistical package for social sciences (SPSS-16) was used for statistical compilation and analysis.

For statistical analysis of difference between groups, independent sample t-test, was applied.

Statistical significance was accepted at $\mathrm{p}<0.05$.

RESULTS: Hematological Parameters of Intravenous iron and oral iron group.

Mean change \pm Sd

(t-test for Equality of Means)

\begin{tabular}{|l|l|l|l|l|}
\hline Parameters & IV iron group & $\begin{array}{l}\text { Oral iron } \\
\text { group }\end{array}$ & $\begin{array}{l}\text { Mean } \\
\text { difference }\end{array}$ & $\begin{array}{l}\text { Sig. } \\
\text { (2-tailed) }\end{array}$ \\
\hline Hb g/dl & $3.162 \pm 0.56$ & $2.0 \pm 0.33$ & 1.15400 & .000 \\
\hline MCV & $8.44 \pm 3.48$ & $2.9 \pm 2.63$ & 5.57800 & .000 \\
\hline S. Ferritin mcg/l & $46.57 \pm 14.12$ & $13.28 \pm 5.31$ & 32.90600 & .000 \\
\hline RBC $\times 1 \mathbf{1 0}^{6} / \mathbf{c u m m}$ & $0.748 \pm 0.39$ & $0.52 \pm 0.25$ & .29440 & .000 \\
\hline MCH & $4.63 \pm 2.08$ & $3.97 \pm 1.75$ & .70600 & .070 \\
\hline $\begin{array}{l}\text { Reticulocyte } \\
\text { count }\end{array}$ & $0.6 \pm 0.33$ & $0.476 \pm 0.204$ & .13200 & .020 \\
\hline MCHC & $5.87 \pm 0.860$ & $5.30 \pm 1.13$ & .57000 & .006 \\
\hline S. Iron $\mathbf{~ m g / d l ~}$ & $91.49 \pm 16.58$ & $99.6 \pm 17.16$ & -8.16400 & .017 \\
\hline TIBC & $67 \pm 17.65$ & $71.46 \pm 16.39$ & 3.86000 & .260 \\
\hline
\end{tabular}




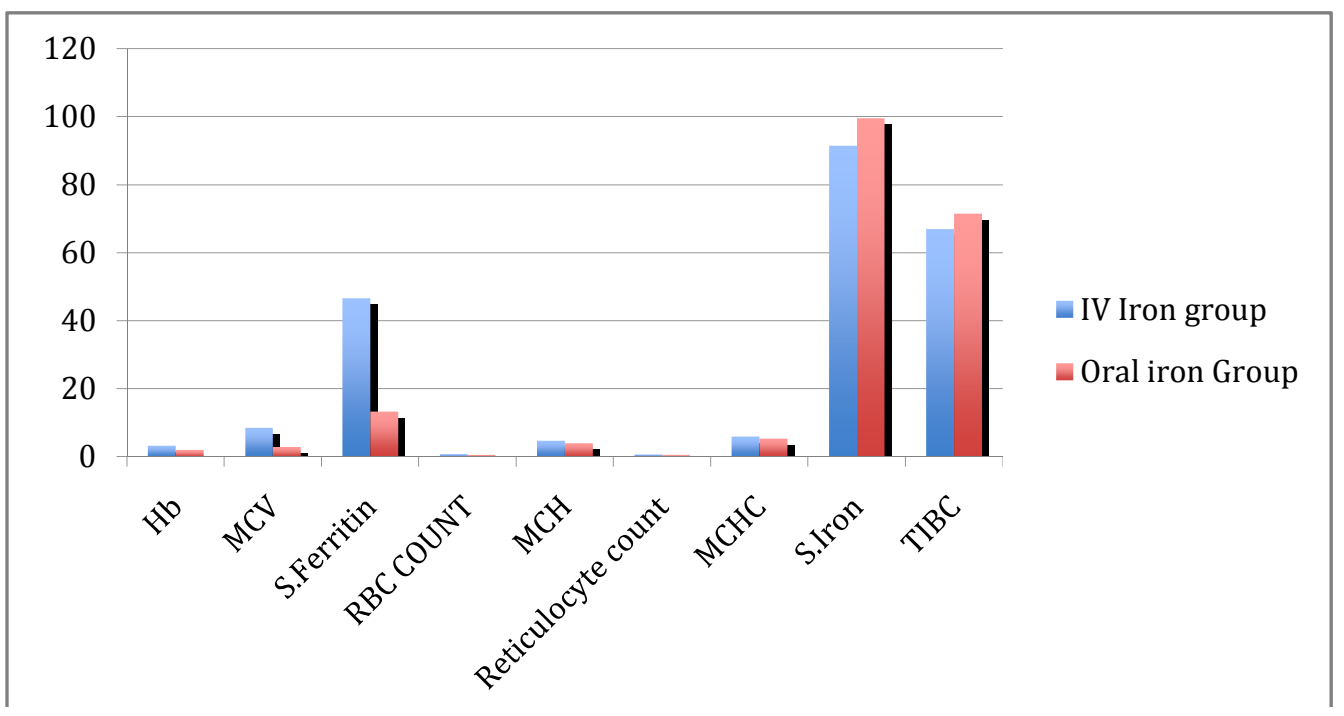

After applying (t-test for equality of means), to find out mean difference between hematologic parameters of Intravenous and oral iron group.

Mean difference of hemoglobin showed a significant improvement $(p=0.000)$ but better outcome in intravenous iron group.

MCV also showed a positive mean difference of 5.57 with significant $\mathrm{p}$ value, and more improvement in IV group.

Serum Ferritin which is the best indicator of iron stores showed a marked improvement in IV iron group with a mean difference of 32.90 and significant $p$ value $(0.000)$.

RBC count had a positive difference of 0.29440 with a significant $p$ value, and better improvement in intravenous group.

MCH also showed better improvement in Intravenous group, but $\mathrm{p}$ value was not significant $(\mathrm{p}=0.70)$.

Serum iron, mean difference had a significant $p$ value of 0.017 .

Mean difference in Reticulocyte count showed a difference of 0.13200 and significant $p$ value (0.020).

MCHC showed a mean difference of 0.57000 and significant $p$ value of $(0.006)$. TIBC, showed significant change with $\mathrm{p}$ value of 0.260 .

DISCUSSION: In this study, the efficacy, safety and tolerability of IVIS in treating pregnancy IDA was compared with OI therapy. IVIS is safe in pregnancy. It corrects anemia at short duration and replenishes iron stores better than OI. This has been the observation in other studies too.(20-22) Comparison with other studies is difficult because of different cut-offs used for lab parameters. OI preparations used are also different. As the rate of increase in hemoglobin is faster, IVIS is suitable for treatment of IDA with lower hemoglobin in the third trimester. There was a highly significant difference in the ferritin level after treatment between the two groups, with iron reserves restored only in the IVIS group, which has also been observed by Bayoumeu et al.(20) Increase in ferritin is not because of direct intravenous injection of iron complex; rather, it is because the IVIS complex releases iron rapidly to endogenous iron binding proteins with no deposition in the parenchymal tissue. It has a half-life of about 6 h.(23) This is an advantage of IVIS over iron dextran or iron gluconate. 
After treatment, the IVIS group maintained hemoglobin with routine supplementation of OI in the present study, unlike in Bayoumeu et al.'s study, where no additional oral supplementation was given. Because of the high prevalence of anemia (57.9\%) in pregnant women as per the National Family Health Survey-3,(24) oral supplementation even with normal iron stores is essential in India. Unlike in the parenteral iron-treated group, once the anemia is corrected with OI, absorption slows down. This is responsible for the iron stores not being replenished with OI, unlike intravenous iron.

The limitations of this study were that, although IVIS increased serum ferritin significantly, Patients were not followed-up in postnatal period to determine whether hemoglobin levels were maintained during lactation because of higher stores. As we did not repeat serum Ferritin in post-natal period due to high cost and lots of follow-up of patients.

So there is still scope for future studies to determine the effect of early diagnosis and correction of anemia not only antenatally but to have sustained hemoglobin levels even in the post-natal period.

We conclude that Oral iron increases hemoglobin comparably with intravenous iron, but does not replenish iron stores as much as IVIS. This is significant in our country where women may become anemic during lactation and might again conceive in lactational amenorrhea, when their iron stores have not been replenished. However oral iron is cheap, but its compliance is poor due to major gastric side effects.

Overall Iron sucrose appears to be a treatment of choice with no serious side effects indicated in the rapid correction of anemia in pregnancy or restoring maternal iron reserves. If used in time this treatment will certainly help to reduce the risk of peri-partum blood transfusion.

As no major adverse effects were noted with Iron sucrose, it is a safe option with good efficacy for the treatment IDA with a narrow side effect profile.

Thus Iron sucrose has revolutionized anemia management in pregnancy with high parity and low resources especially in developing countries like ours.

\section{REFERENCES:}

1. DeMayer EM, Tegman A. Prevalence of anemia in the World. World Health Organ Qlty 1998; 38:302-16.

2. WHO.2004.Micronutrient deficiency: Battling iron deficiency anaemia: the challenge.Available from:http://www.who.int/nut/ida.htm,accessed on April 24, 2008.

3. Malhotra J. Iron deficiency anemia in India.FOGSI Focus 2009; 9-11.

4. Raja KS, Janjua NB, Khokhar N. Intravenous iron sucrose complex therapy in iron deficiency anemia in the pregnant women.Rawal Med J.2003;28: 40-3.

5. World Health Organization. Nutritional anaemias. Tech Rep Ser 1972; 503.

6. CDC criteria for anemia in children and child bearing aged women.MMWR Morb Mortal Wkly Rep 1989; 38: 400-4.

7. Bernard BJ, Hakimi M, Palletier D.An analysis of anemia and pregnancy - related maternal mortality .J Nutr 2001; 131:604S-615S.

8. Murray CJL, Lopez AD. Global and regional causes of death patterns in 1990.Global comparative assessments in health sector-Disease burden, expenditures and intervention packages. 1994: 21-54, WHO Geneva, Switzerland.

9. WHO. The prevalence of anemia in women: a tabulation of available information $1992 \mathrm{~b}$ Maternal health and safe motherhood programme.Geneva, Switzerland. 
10. WHO (1996) Revised 1990 estimate of maternal mortality. A new approach by WHO and UNICEF.WHO, Geneva, Switzerland.

11. Bhatt R. Maternal mortality in India, FOGSI WHO study. J Obstet gynecol Ind 1997;47:207-214.

12. Scholl TO, Hediger, ML, Fischer RL, Shearer JW Anaemia vs iron deficiency:increased risk of preterm delivery in a prospective study. Am J Clin Nutr, 1992; 55: 985-988.

13. Godfrey KM, Redman CWG, Barker DJP, Osmond C The effect of maternal anemia and iron deficiency on ratio of fetal weight to placental weight. Br J Obstet Gynaecol, 1991;98:886-891.

14. Barker DJP, Bull AR, Osmond C, Simmonds SJ.Fetal and placental size and risk of hypertension in adult life. Br Med J 1990;301:359-262.

15. Institute of Medicine, Committee on Nutritional status during pregnancy and lactation .Nutrition during pregnancy. Washington DC : National academy press; 1990:272-98.

16. Wali A, Mushtaq A, Nilofer. Comparative study: efficacy, safety and compliance of intravenous iron sucrose and intramuscular iron sorbitol in iron deficiency anemia of pregnancy. J Pak Med Assoc.2002; 52: 392-5.

17. Bhandal N, Russell R. Intravenous versus oral iron therapy for postpartum anemia.BJOG. 2006; 113:1248-52. Doi:10.1111/j.1471-0528.2006.0162.x.

18. Giannoulis C, Daniilidis A, Tantanasis T, et al. Intravenous administation of iron sucrose for treating anemia in postpartum women. Hippokratia. 2009; 13:38-40.

19. 19.. Fernando Arias : Practical guide to high risk pregnancy and delivery. 3 rd edition pg 466-70.

20. Bayoumeu F, Subiran-Buisset C, Baka NE, Legagneur H, Monnier-Barbarino P, Laxenaire MC. Iron therapy in iron deficiency anemia in pregnancy: Intravenous route versus oral route. Am J Obstet Gynecol. 2002;186:518-22. [PubMed]

21. al-Momen AK, al-Meshari A, al-Nuaim L, Saddique A, Abotalib Z, Khashogji T, et al. Intravenous iron sucrose complex in the treatment of iron deficiency anemia during pregnancy. Eur J Obstet Gynecol Reprod Biol. 1996;69:121-4. [PubMed]

22. Al RA, Unlubilgin E, Kandemir O, Yalvac S, Cakir L, Haberal A. Intravenous versus oral iron for treatment of anemia in pregnancy: a0 randomized trial. Obstet Gynecol. 2005;106:1335-40. [PubMed]

23. Silverstein SB, Rodgers GM. Parenteral iron therapy options. Am J Hematol. 2004;76:748. [PubMed]

24. National Family Health Survey 3. India 2005-06; International Institute of Population Sciences, Mumbai, India and ORC Macro, Calverton, Maryland, USA. 2007 Oct 